\title{
ORAL AND DENTAL MANIFESTATIONS OF XEROSTOMIA: PUBLIC HEALTH IMPLICATIONS OF A COMMON AND UNDER-RECOGNISED ADVERSE DRUG REACTION
}

\author{
Mark Schifter \\ Staff Specialist and Head, Oral Medicine Unit \\ Department of Oral Diagnostic Sciences \\ Westmead Centre for Oral Health \\ (formerly Dental Clinical School, Westmead Hospital)
}

The use of medications that have significant adverse implications for oral health and the safe delivery of dental care is increasing. ${ }^{1}$ Medication use in general is expected to rise as the population ages, as will the number of dentate patients in the over-65 age group. ${ }^{1}$ Adverse drug reactions, the most common of which is xerostomia, or dry mouth, may have direct effects on oro-dental structures. ${ }^{1,2}$ Decreased salivary flow and function leads directly to increased dental decay (caries) and impairment of the integrity of the oral lining, and it compromises such functions as tasting, speaking and swallowing. ${ }^{3,4}$ As described in the article by Barbara Anne Taylor, these effects have broader public health implications. Poor oral health status has been linked recently to major systemic conditions, such as cardiovascular disease. ${ }^{5,6}$ As well, diabetes and infective endocarditis have a wellestablished association with dental disease. ${ }^{7,8}$ This article examines the major groups of drugs causing xerostomia and the potential impact on both oral and general health.

Xerostomia is a symptom and does not itself represent a pathological entity. It may be defined as dryness of the oral mucosa of varying severity and a reduced flow in saliva (salivary hypofunction). ${ }^{9}$ Up to 1.5 litres of this complex secretion is produced every 24 hours. Of this, 20 per cent is produced by the parotid glands, which increase their contribution by up to 50 per cent on stimulation; 65 per cent by the submandibular glands; and the remaining 15 per cent by the sublingual and minor salivary glands. ${ }^{9}$

Little is known of the prevalence or the severity of salivary hypofunction in Australia. In the United States, surveys have found that up to 60 per cent of institutionalised elderly people and 30 per cent of older community-dwelling individuals complain of a dry mouth. ${ }^{10,11}$

The leading causes of xerostomia are iatrogenic, predominantly as an adverse side-effect of many medications. Although the exact mechanisms are not understood, up to 400 drugs have been shown to induce xerostomia. $^{1-4,12}$ The main classes of drugs implicated include the anticholinergics; the anti-hypertensives, especially the diuretic agents; the anti-depressive agents, particularly the tricyclics; and most analgesics. These classes of drugs are used predominantly, often in combination, by older Australians. Aging itself is associated with some diminution of salivary function; however, this physiological change is much less severe than previously thought. Another group of drugs that cause profound salivary hypofunction are the major antipsychotics, especially the phenothiazines and lithium. ${ }^{12}$

The effects of reduced salivary flow are potentially profound. Loss of the essential lubricating quality of saliva results in impairment of speech, mastication, and the early phases of swallowing and digestion. One study demonstrated that some 70 per cent of members of a retirement village suffered from medication-induced xerostomia that led directly to their avoidance of foods that were drier and more difficult to masticate and swallow, such as bread and crunchy vegetables. Concern was expressed that this could contribute to under-nutrition in these populations. ${ }^{9}$

Saliva is essential for protection against dental caries. Saliva contains anti-bacterial substances that inhibit and destroy cariogenic (caries-causing) members of the plaque flora. Saliva buffers the acid produced by cariogenic bacteria that can lead to dental decay by dissolving the mineralised-calcified component of the teeth. As well, saliva is essential for the re-mineralisation process, which allows the early repair of non-cavitated carious lesions, effectively forestalling decay. Thus, lack of saliva can lead to the loss of teeth by dental decay. ${ }^{3}$

Replacing lost teeth with dental prostheses in xerostomia patients is unsatisfactory. Dentures are expensive to manufacture and are highly reliant on the lubricating properties of saliva for their retention and function. Further, they exacerbate the problem of decay by retaining food as well as the plaque containing the bacteria that process food debris into the acid responsible for dental decay.

Until recently, the morbidity and poorer quality of life associated with xerostomia has been limited to the resultant need to extract teeth and fit replacement prostheses. However, new evidence demonstrates that poor oral health may be linked to adverse systemic conditions such as coronary heart disease (CHD). ${ }^{5,6}$ A recent study demonstrated a statistically significant association between the diagnosis of CHD and such oral health parameters as the number of missing teeth and complaints of xerostomia. Indeed, these oral health parameters were more strongly associated with CHD than such recognised risk factors as elevated serum cholesterol levels, obesity, late-onset diabetes and tobacco use. ${ }^{5}$ The relationship between these oral health parameters and CHD is not 
understood, but one hypothesis is that certain oral flora may play a role in the pathogenesis of CHD. Consequently, the risks associated with xerostomia may be more extensive than previously believed.

Therefore, while the management of xerostomia is highly problematic, it is very important. Education of general medical practitioners and community-based pharmacists regarding the detrimental and xerogenic effect of certain medications is vital. Patients should be advised of the effect of their medications; warned to avoid simple potentially cariogenic sugars, such as table sugar; and encouraged to use non-calorific sweeteners, improve oral hygiene practices and attend their dentist for review.

For specific cases of salivary hypofunction, treatment is aimed at relief of symptoms. The use of salivary stimulants such as pilocarpine has not been well studied in patients with medication-induced xerostomia. Artificial salivary replacements are by and large unsuccessful. They are poorly tolerated by patients and have none of the antibacterial and re-mineralising abilities of normal saliva. Intensive fluoride preparations are useful for limiting the severity and extent of dental caries; however, they are dependant upon dedicated and regular home use. Topical anti-microbials or specific anti-fungals may also be useful for gingival and mucosal care.

In the absence of Australian research, targeted epidemiological research, in combination with education programs for the professional and lay carers - particularly those responsible for the institutionalised elderly and those with major psychoses — should be a public health priority. The morbidity, potential major systemic health effects, and the social and financial costs of xerostomia with its resultant loss of function and oral comfort need to be more fully researched and publicised. What has been learned from the preventive reforms put in place for the younger members of our community, such as the benefits of water flouridation, needs now to be re-assessed and applied to the increasing number of older Australians.

\section{REFERENCES}

1. Smith RG, Burtner AP. Oral side-effects of the most frequently prescribed drugs. Special Care Dent 1994; 14: 96-101.

2. Lewis IK, Hanlon JT, Hobbins MJ, Beck JD. Use of medications with potential oral adverse drug reactions in community-dwelling elderly. Spec Care Dent 1993; 13: 171176.

3. Papas AS, Joshi A, MacDonald SL, et al. Caries prevalence in xerostomic individuals. J Can Dent Assoc 1993; 59: 171179.

4. Atkinson JC, Wu AJ. Salivary gland dysfunction: Causes, symptoms, treatment. J Am Dent Assoc 1994; 125: 409-416.

5. Loesche WJ, Schork A, Terpenning MS, et al. Assessing the relationship between dental disease and coronary heart disease in elderly US veterans. $J$ Am Dent Assoc 1998; 129: 301311.

6. Beck J, Garcia R, Heiss G, Vokonos PS, Offenbacher S. Periodontal disease and cardiovascular disease. J Periodontal 1996; (Suppl) 67: 112-137.

7. Finney LS, Finney MO, Gonzalez-Campoy JM. What the mouth has to say about diabetes. Postgrad Med 1997; 102 : 117-124, 126.

8. Strom BL, Abrutyn E, Berlin JA, et al. Dental and cardiac risk factors for infective endocarditis: A population-based, case control study. Ann Int Med 1998; 129: 761-769.

9. Crockett DN. Xerostomia: The missing diagnosis. Aust Dent $J$ 1993; 38: 114-118.

10. Marcus SE, Kaste LM, Brown LJ. Prevalence and demographic correlates of tooth loss among the elderly in the United States. Spec Care Dent 1994; 14: 123-127.

11. Loesche WJ, Bromberg J, Terpenning MS, et al. Xerostomia, xerogenic medications and food avoidances in selected geriatric groups. J Am Geriatr Soc 1995; 43: 401-407.

12. Peeters FPML, deVries MW, Vissink A. Risks for oral health with the use of antidepressants. Gen Hosp Psychiatry 1998; 20: $150-154$.

The Public Health Bulletin wishes to acknowledge the work of the oral health editorial committee in producing this series on oral health. Members of the committee are:

Patrick Meaney, Senior Vice-President, NSW Branch of the Australian Dental Association

Barbara Taylor, Head of the Periodontics Department, United Dental Hospital Richard Widmer, Head of the Department of Dentistry, New Children's Hospital David Wright, Director of Dental Services, Hunter Area Health Service Jane Bell, Policy Analyst, Oral Health Branch, NSW Health Department Alan Patterson, Chief Dental Officer, Oral Health Branch, NSW Health Department. Mary Osborn also contributed to the series during her Public Health Officer placement in the Oral Health Branch. In particular, we thank Jane Bell who has played a significant role in coordinating the series. 